\title{
Trim the gut, lose the weight - and the bone
}

\author{
Soravis Osataphan and Mary Elizabeth Patti
}

Research Division, Joslin Diabetes Center, and Harvard Medical School, Boston, Massachusetts, USA.

\begin{abstract}
Vertical sleeve gastrectomy (VSC) is an effective therapeutic approach for obesity and type 2 diabetes but is associated with osteoporosis. In this issue of the $J C I$, Li et al. report that VSC rapidly reduces bone mass, as observed in humans, via rapid demineralization and decreased bone formation, independent of weight loss or $\mathrm{Ca}^{2+} /$ vitamin D deficiency. VSG also reduces bone marrow adipose tissue, in part via increased granulocyte-colony stimulating factor (G-CSF). The interplay between VSG-mediated effects on systemic metabolism and bone biology remain to be investigated. These findings suggest novel mechanisms and therapeutic targets for bariatric surgery-induced osteoporosis.
\end{abstract}

because these subpopulations are regulated independently of peripheral adipose tissue and have distinct developmental and transcriptional lineages (5). Hematopoietic lineages were also modified early after VSG, with increased myeloid cells and reduced erythroid cells.

\section{Mechanisms underlying the effects of bariatric surgery on bone loss}

Which mechanisms mediate these diverse effects of bariatric surgery on bone and the marrow niche? Li and colleagues hypothesized that granulocyte-colony stimulating factor (G-CSF) might contribute to the robust increase in myeloid-lineage cells in the marrow and bone loss after VSG. Indeed, G-CSF was markedly increased as early as one week after VSG surgery in mice, with more modest increases observed in a human cohort. The mechanisms responsible for increased G-CSF remain uncertain. Experimental increases in G-CSF stimulated myelopoiesis, as expected, while also reducing bone marrow adipocytes and bone mass. Finally, the effects of VSG on bone marrow phenotypes were reduced in G-CSF-null mice. However, bone mass was still reduced after VSG in G-CSF-null mice, indicating that other factors are required for VSG-induced bone loss.

What additional factors modulated in response to intestinal surgery might contribute to bone loss and changes in the marrow niche? After both VSG and RYGB, ingested food rapidly exits the revised stomach pouch. This accelerated delivery of undigested food to the intestine yields pleiotropic effects on intestinal cell populations, secretion of metabolically active peptides, increases in bile acids, changes in the microbiome, and altered absorption of glucose and other nutrients - all potential mediators of the so-called gut-brainliver axis regulating appetite and systemic metabolism (6). Li et al. demonstrate that weight loss, changes in calcium metabolism (at least those discernible from plasma measurements), and the intestinally derived hormones GLP1/2 are not 


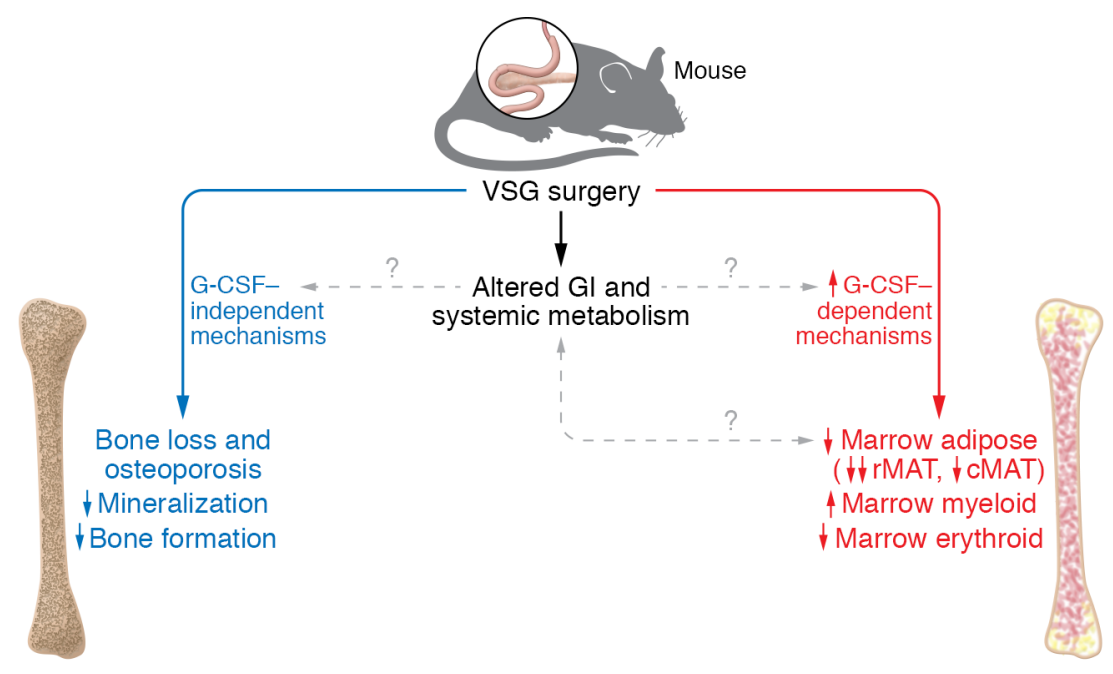

required for the profound bone loss occurring early after VSG. However, many additional candidates could contribute to bone metabolic effects. These could be primary (resulting directly from changes in intestinal anatomy) or secondary (resulting from changes in systemic metabolism). For example, intestinally derived hormone peptide YY, which is induced after bariatric surgery, has been linked with increased bone turnover in mice (7). By contrast, the glucose-dependent insulinotropic polypeptide (8), also increased after surgery, can inhibit markers of bone resorption (9). The gut microbiome can also regulate bone mass; thus, bacterial species or their metabolic byproducts altered in response to VSG may be an important missing link in this paradigm (10). Changes in bile acid levels or metabolism, downstream pathways stimulated by bile acids, and other metabolites could contribute to functional changes in bone and or marrow adipocyte populations $(11,12)$.

Beyond the gastrointestinal tract, bariatric surgery induces secondary changes in other metabolically active organs such as muscle and pancreatic islets. Interestingly, a muscle-derived hormone, irisin, is increased after both bariatric surgery and exercise, and can modulate bone mineralization $(13,14)$. Thus, irisin could mediate a muscle-bone axis altered after bariatric surgery. Likewise, plasma levels of G-CSF are increased after exercise, potentially from muscle (15). Bariatric surgery and weight loss improve whole-body insulin sensitivity, with reduced fasting insulin levels. Because insulin is anabolic for bone, it would be interesting to investigate the contribution of insulin and other growth factors to bone remodeling after bariatric surgery $(16,17)$.

\section{Bone marrow adipocytes and bariatric surgery-mediated bone loss}

A distinct role for bone marrow adipocytes remains a possibility. VSG induces a major loss of bone marrow adipocyte populations, particularly from the so-called regulated BMAT and less so from the constitutive BMAT. BMAT is an important source of the insulin sensitizer adiponectin. Although bone marrow adipocyte loss was not observed in humans undergoing bariatric surgery (2), this could reflect less sensitive methodologies in human studies. Paradoxically, recent studies have demonstrated a crucial role for the expansion of marrow adipocytes during caloric restriction (18), potentially via BMAT-derived adiponectin. Thus, future studies examining the impact of bone marrow adipocytes in mediating the systemic metabolic and bone effects of bariatric surgery will be critical for our understanding of bariatric surgery.

\section{Unanswered questions}

While the data of Li et al. add much to our understanding, many questions remain. What mechanisms and tissues are responsible for increases in G-CSF after VSG? Are mechanisms underlying early post-bariatric surgery bone loss conserved from rodents to humans? Do these same mechanisms apply to osteoporosis occurring late after bariatric surgery in humans? What is the impact of
Figure 1. VSC induces bone loss by reducing mineralization and bone formation. VSGinduced bone loss (left). In parallel, G-CSF decreases bone marrow adipose tissue and activates myeloid proliferation (right). The contribution of altered gastrointestinal physiology and systemic metabolism to both bone loss and increased G-CSF remain to be investigated.

vitamin D deficiency, which is common in obese individuals preoperatively and at late postoperative periods? Could tissue-level vitamin D deficiency or cellular resistance contribute to impaired osteoid formation in the early postoperative state? Since increased myeloid and reduced erythroid populations emerge in parallel after VSG, which of these play a primary pathogenic role?

In summary, the study by Li et al. adds to our understanding of the alterations in bone structure and function following VSG. Future studies are needed to define the molecular mediators of bone loss and marrow niche remodeling and to assess the contribution of BMAT to metabolic response to bariatric surgery. Further understanding of these factors will provide crucial information to guide optimal osteoporosis prevention and treatment strategies in this population. Moreover, identification of factors mediating metabolic improvement after bariatric surgery may aid in the design of bariatric mimetics for obesity and T2D.

\section{Acknowledgments}

MEP gratefully acknowledges research grant support from U01 DK114156 (ARMMS-T2D), R01 DK106193, R21 HD091974, and DRC P30 DK036836. SO gratefully acknowledges fellowship support from the Prince Mahidol Award Foundation under the Royal Patronage.

Address correspondence to: Mary Elizabeth Patti, 1 Joslin Place, Room 620, Boston, Massachusetts 02215, USA. Phone: 617.309.2635; Email: mary.elizabeth.patti@ joslin.harvard.edu. 
1. Stein EM, Silverberg SJ. Bone loss after bariatric surgery: causes, consequences, and management. Lancet Diabetes Endocrinol. 2014;2(2):165-174.

2. Bredella MA, Greenblatt LB, Eajazi A, Torriani M, Yu EW. Effects of Roux-en-Y gastric bypass and sleeve gastrectomy on bone mineral density and marrow adipose tissue. Bone. 2017;95:85-90.

3. Yu EW, et al. Effects of gastric bypass and gastric banding on bone remodeling in obese patients with type 2 diabetes. JClin Endocrinol Metab. 2016;101(2):714-722.

4. Li Z, et al. G-CSF partially mediates effects of sleeve gastrectomy on the bone marrow niche. JClin Invest. 2019;129(6):2404-2416.

5. Scheller EL, Cawthorn WP, Burr AA, Horowitz MC, MacDougald OA. Marrow adipose tissue: trimming the fat. Trends Endocrinol Metab. 2016;27(6):392-403.

6. Seeley RJ, Chambers AP, Sandoval DA. The role of gut adaptation in the potent effects of multiple bariatric surgeries on obesity and diabetes.
Cell Metab. 2015;21(3):369-378.

7. Wong IP, et al. Peptide YY regulates bone remodeling in mice: a link between gut and skeletal biology. PLOS ONE. 2012;7(7):e40038.

8. Mahendran Y, et al. Association of ketone body levels with hyperglycemia and type 2 diabetes in 9,398 Finnish men. Diabetes. 2013;62(10):3618-3626.

9. Christensen MB, et al. Glucose-dependent insulinotropic polypeptide (GIP) inhibits bone resorption independently of insulin and glycemia. J Clin Endocrinol Metab. 2018;103(1):288-294.

10. Ohlsson C, Sjögren K. Effects of the gut microbiota on bone mass. Trends Endocrinol Metab. 2015;26(2):69-74.

11. Id Boufker H, et al. Role of farnesoid X receptor (FXR) in the process of differentiation of bone marrow stromal cells into osteoblasts. Bone. 2011;49(6):1219-1231.

12. Cho SW, et al. Positive regulation of osteogenesis by bile acid through FXR. J Bone Miner Res. 2013;28(10):2109-2121.
13. Lee YJ, et al. Association of circulating irisin concentrations with weight loss after Roux-en-Y gastric bypass surgery. Int J Environ Res Public Health. 2019;16(4):E660.

14. Kim H, et al. Irisin mediates effects on bone and fat via $\alpha \mathrm{V}$ integrin receptors. Cell. 2018;175(7):1756-1768.e17.

15. Yamada M, Suzuki K, Kudo S, Totsuka M, Nakaji S, Sugawara K. Raised plasma G-CSF and IL-6 after exercise may play a role in neutrophil mobilization into the circulation. JAppl Physiol. 2002;92(5):1789-1794

16. Fulzele K, et al. Insulin receptor signaling in osteoblasts regulates postnatal bone acquisition and body composition. Cell. 2010;142(2):309-319.

17. Ferron $\mathrm{M}$, et al. Insulin signaling in osteoblasts integrates bone remodeling and energy metabolism. Cell. 2010;142(2):296-308.

18. Cawthorn WP, et al. Bone marrow adipose tissue is an endocrine organ that contributes to increased circulating adiponectin during caloric restriction. Cell Metab. 2014;20(2):368-375. 